\title{
The Pathogenetic Background of Transfusion-Associated Acute Lung Injury Cannot Be Confined to the Passive Transfer of Donor Leucocyte Antibodies to Transfusion Recipients
}

\author{
Hans-Gert Heuft ${ }^{\mathrm{a}} \quad$ Walter Hitzler ${ }^{\mathrm{b}}$ \\ a Institute for Transfusion Medicine, Hanover Medical School, \\ ${ }^{b}$ Transfusion Centre, Clinics of Johnannes Gutenberg University Mainz, Germany
}

When considering severe life-threatening transfusion complications, the transfusion-associated acute lung injury (TRALI) syndrome is currently the most outstanding challenge to transfusion medicine. Several haemovigilance systems in the UK, in France and in the USA identified TRALI to be the most frequent cause of transfusion-associated death, even if cases of ABO mistransfusion are included [1]. Data from the UK, accumulated 1996 through 2005 by the British Serious Hazards of Transfusion (SHOT) initiative, reported on 38 cases of transfusion-associated death at least possibly due to TRALI [2]. According to SHOT, for the observation period 1999 through 2005 the residual risk for components implicated in severe TRALI with an imputability level of 'highly likely/probably' was 1:76,000 for plasma-rich components (e.g. fresh frozen plasma (FFP) and platelet concentrates (PC)) and 1:440,000 for plasma-poor components (e.g. red cell concentrates (RCC) in additive solution) [3]. The French Health Products Safety Agency registered 10 transfusion-associated deaths at least 'probably' attributable to TRALI from 2002 to 2005 [4]. The American Red Cross TRALI surveillance study revealed 38 fatalities of probable TRALI between 2003 and 2005 [5]. For the USA, the risk of TRALI in transfusion recipient fatalities was calculated to be in the range of 1:2.5 Mio for RCC transfusion, 1:203,000 for FFP transfusion, 1:321,000 for apheresis PC transfusion and 1:2.1 Mio for whole blood-derived pooled PC transfusion. In Germany, an intensified surveillance study was performed by the Paul Ehrlich Institute (PEI) from January 2006 to July 2007. During this period 31 severe TRALI cases with 7 fatalities were observed.

Of note, in the majority of transfusion recipients with severe TRALI, females with a history of at least one previous pregnancy (many of them with detectable antibodies to leucocyte antigens (LC-AB)) were identified as donors of the implicated blood components. This was a uniform finding in haemovigi- lance systems and TRALI studies as demonstrated by data from the SHOT initiative (2003-2005 41 TRALI cases highly likely or probable; 29/41 LC-AB-positive females (70\%)), the ARC surveillance study (2003-2005 38 fatalities due to TRALI; 27/38 LC-AB-positive females (71\%)) or the PEI surveillance study (2006-2007 31 TRALI cases highly likely; 21/31 LC-AB-positive females $(68 \%))$.

In view of these compelling data, it cannot surprise that preventive measures have been already taken (UK, Switzerland) or are lively discussed (Germany, USA) to restrain female donors with a history of pregnancy from plasma donation, to exclude plasma from females with pregnancy history from quarantine plasma production or to disperse whole blood-derived platelets for pooled PC predominantly in male plasma. In Germany, several German Red Cross (GRC) and university clinic blood donation services have started to test large cohorts of females with a history of pregnancy for HLA as well as HNA antibodies [6-8].

In this situation one might wonder why the organizers of the StKB-Zukunftsworkshop Hämo- und Zelltherapie have found it necessary to hold an international symposium on TRALI. In our view the pathogenetic background of TRALI is not as clear as haemovigilance and surveillance study data suggest. For instance, the outstanding role of LC-AB as a decisive trigger for TRALI can be easily constrained by simple calculations on the frequency of transfusions of plasma-rich blood components containing LC-AB. For Germany, we have based such calculations on the following assumptions and, if possible, on existing data: According to the announcements to the PEI under the terms of $\S 21$ TFG [9], the number of FFP, definitely transfused to patients, has a stable level around 1.0 Mio transfused units (TU) per year (e g. 2001 1.067 Mio TU; 2002 1.052 Mio TU; 2003 1.049 Mio TU; 2004 0.988 Mio TU), whereas the number of PC transfusions was increasing from

\begin{tabular}{ll}
\hline KARGER & @ 2008 S. Karger GmbH, Freiburg \\
$\begin{array}{l}\text { Fax +497614520714 } \\
\begin{array}{l}\text { E-mail Information@Karger.de } \\
\text { www.karger.com }\end{array}\end{array}$ & $\begin{array}{l}\text { Accessible online at: } \\
\text { www.karger.com/tmh }\end{array}$
\end{tabular}


Table 1. Transfusion of plasma-rich blood components (FFP, PC) containing LC-AB

\begin{tabular}{lll}
\hline & $\begin{array}{l}\text { Germany } \\
1992-2006\end{array}$ & $\begin{array}{l}\text { Hanover Medical } \\
\text { School 2006 }\end{array}$ \\
\hline Number of plasma-rich blood components & $18,000,000$ & 36,200 \\
Number of donors & n.a. & 24,300 \\
Proportion of females, \% & 47 & 47 \\
Females with previous pregnancies, \% & 59 & 59 \\
Females with previous pregnancies and LC-AB, \% & 9 & 9 \\
Number of transfusions with LC-AB per year & 449,200 & 607 \\
\hline
\end{tabular}

n.a. $=$ Not available
0.266 Mio (in 2001) to 0.321 Mio TU (in 2004). As the registration rate for transfusions to patients varies from 60 to $70 \%$ per year, a total number of 1.2 Mio transfusions of plasmarich blood components per year appears to be a very conservative calculation base. For Germany, no reliable data exist for the proportion of female blood donors with a history of at least one pregnancy. However, we have found one large study who evaluated the proportion of female WB and PC donations with previous allo-exposure (pregnancies and blood transfusion) out of 6 large geographical areas in the USA for the year 2006 [10]. Related to a total number of 1.02 Mio whole blood / PC donations, the authors found a female donation rate of $47 \%$ (0.476 Mio female donations) $2.2 \%$ of which had a history of blood transfusion and $59 \%$ of which had at least one previous pregnancy. The latter number fits well to results of an investigation on the attitude of women in Germany to children and childlessness that revealed a rate of $35 \%$ childlessness in women beyond 35 years of age [11]. There are two German studies that allow a relatively reliable estimation of the proportion of LC-AB carriers among previously pregnant female blood donors. The GRC Blood Donation Services Hagen and Bad Kreuznach investigated 2,752 female donors with $\geq 1$ pregnancy and 1,120 females with $\geq 2$ pregnancies. The rate of LC-AB carriers with HLA class I antibodies was 7.8 or $9.6 \%$. Either alone or additionally to the HLA class I antibodies, a number of women had HLA class II antibodies (45/812 women; $5.5 \%$ ), and a small proportion of HNA antibody carriers (16/2,752 women; $0.6 \%$ ) was also detected. For the purpose of our calculations a LC-AB carrier rate of $9 \%$ was assumed as a realistic value. Based on these assumptions and data, we calculated the number of transfusions of LC-AB-containing plasma-rich blood components for Germany for the period 1992 through 2006 and for Hanover Medical School for the year 2006; the results are given in table 1 . It must be pointed out that the calculated numbers of 449,000 transfused plasma-rich blood components for Germany for the 15-year period 1992-2006 represent least numbers as roughly $30 \%$ of institutions that apply transfusions do not announce them to the PEI. Moreover, approximately 600 LC-AB-containing plasma-rich blood components must have been applied at Hanover Medical School in 2006, but no TRALI cases were observed.
One might argue that the rare coincidence between transfusion of a LC-AB-containing plasma-rich blood components and TRALI must be due to the rare coincidence of a LC-AB and its cognate antigen in the patient. However, for several reasons this is also no convincing objection. First, most LC$\mathrm{AB}$ carriers usually have several rather than only one LC-AB. Second, despite the high polymorphism that characterizes the HLA system, many important HLA antigens show phenotype frequencies above $10 \%$ (e.g. HLA-A1, HLA-B7, HLA-DR11 and others) or even far above $10 \%$ (e.g. HLA-A2). Third, in contrast to the HLA system, the HNA system shows a low polymorphism [12], and most HNA antigens are widely spread in the general population (e.g. HNA-1a approximately $55 \%$, HNA-3a $>90 \%$ ). Thus, the passive transfer of LC-AB via a plasma-rich blood component into a patient with a cognate antigen cannot be a rare event. This view is supported by a number of look-back studies from Poland [13] and the USA $[14,15]$. These studies were prompted by single cases of severe TRALI ('index cases'); each of them was caused by longterm female blood donors with a history of blood donation of up to 16 years. In these donors LC-AB were detected when the diagnosis 'TRALI' in the index case was made. As shown in table 2, over the years these donors had given a total 623 blood components. Of these, the majority $(322 / 623 ; 52 \%)$ were plasma-rich blood components. In the study of Toy et al. [15], HLA typing of the transfusion recipients was available in 55 of 103 patients. As the donor of this study was broadly sensitized, 54 of the 55 transfusion recipients showed at least one cognate antigen (and many of these recipients presented even several cognate antigens), but no TRALI. In the study of Maślanska et al. [13] out of a group of 62 LC-AB-positive female donors with a total of 296 blood products, one RCC produced severe TRALI [13]. The donor of the RCC had 12 additional transfusion recipients without TRALI. From 26 of the 61 polish female donors without evidence of TRALI in transfusion recipients, HLA typing results of transfusion recipients and specific HLA antibodies in the donors could be retrieved. In 11 of these 26 donor-transfusion recipient pairs (42\%), a cognate HLA antigen in the recipient was demonstrable, but no TRALI.

What can we learn from these calculations and look-back study results? Many case reports are suggestive for LC-AB to 
Table 2. Results of look-back studies after TRALI reactions

\begin{tabular}{llll}
\hline & $\begin{array}{l}\text { Maślanska et al., } \\
2007[13]\end{array}$ & $\begin{array}{l}\text { Win et al., } \\
\text { 2002 [14] }\end{array}$ & $\begin{array}{l}\text { Toy et al., } \\
\text { 2004 [15] }\end{array}$ \\
\hline Number of donors with LC-AB & 62 & 6 & 1 \\
BC transfused & 296 & 141 & 186 \\
RCC & 187 & $16^{*}$ & 0 \\
PC & 61 & $17^{*}$ & 179 \\
FFP & 48 & $10^{*}$ & 7 \\
Number of patients transfused, n & n.a. & n.a. & 55 \\
Number of patients available for with HLA/HNA typing, n & 26 & n.d. & \\
Number of patients with cognate HLA or HNA antigen(s), & & & $54(98 \%)$ \\
$\quad$ but no TRALI (\%) & $11(42 \%)$ & n.d. & \\
\hline
\end{tabular}

n.a. = Not available; n.d. $=$ not determined.

*Related to a subgroup of 43 blood components donated within a period of 5 years before the index TRALI case play a role as a trigger for a severe TRALI reaction, but their significance in the pathogenesis of TRALI in general is greatly overestimated! This is even true in many (if not most) examples of a coincidental concourse of specific HLA/HNA antibodies and their cognate antigen(s) in a transfusion recipient. The pathogenetic background of TRALI is complex and cannot be confined to the passive transfer of donor LC-AB to transfusion recipients. Other pathogenetic factors are also involved, and their role still remains to be determined!

It was this kind of perception that prompted the Organizing Committee of the 2nd StKB-Zukunftsworkshop Hämo- und Zelltherapie to hold an international symposium on TRALI in Mainz, Germany, on November 8, 2007. The purpose of this symposium was to give an overview on current concepts on the pathophysiology and clinical aspects of TRALI (Part I) and to present the latest data on the epidemiology of TRALI among transfusion recipients in Europe (Part II). This editorial cannot describe all highlights of the symposium. However, in a few sentences some controversies around TRALI become clear. In Part I, the two outstanding researchers of the TRALI scene, Mark A. Popovsky from Harvard Medical School and Christopher C. Silliman from the School of Medicine of the University of Colorado, presented their opposing concepts of
'Immune TRALI' versus 'Non-Immune TRALI'. In Part II, the UK haemovigilance data (SHOT initiative, presented by Catherine E. Chapman) and the French haemovigilance data (e-FIT, presented by Philippe Renaudier) showed surprisingly different patterns with respect to the main TRALI-causing blood components. The SHOT group identified FFP as main source contributing to TRALI. This finding formed the base for the gradual stepwise exclusion of female plasma from quarantine FFP and PC production in the UK since 2003 ('male only' plasma policy). In contrast, in the French haemovigilance data PC and RCC were the predominant blood components causing TRALI so that in France a 'male only' plasma policy is currently not considered as a useful prevention strategy against TRALI.

To conclude, the 2nd StKB-Zukunftsworkshop Hämo- und Zelltherapie has clearly shown that neither the pathogenetic nor the epidemiological background of TRALI is fully understood. More than ever TRALI appears as a multifactor transfusion reaction that needs multifactor solutions. The simple exclusion of females with a history of previous pregnancies is not justified and rather will create additional problems in transfusion medicine than eliminate this complication.

\section{References}

1 Bux J: Transfusion-related acute lung injury (TRALI): a serious adverse event of blood transfusion. Vox Sang 2005;89:1-10.

2 Serious Hazards of Transfusion (SHOT). Annual Report 2006. www.shot.org.

3 Chapman CE: National Blood Service TRALI reduction policies: implementation and effect. Transfus Med Hemother 2008:35(2):89-92.

4 Willaert B. Willaert B: French haemovigilance data on platelet transfusion. Presentation of the Agence Francaise de Securite Sanitaire des Produits de Sante (AFSSAPS). Symposium of Platelet Usage and Platelet Product Selection, Hannover, Germany, June 18, 2007. http//afssaps.sante.fr.

$\checkmark 5$ Eder AF, Herron R, Strupp A, Dy B, Notari EP Chambers LA, Dodd RY, Benjamin RJ: Transfusion-related acute lung injury surveillance (20032005) and the potential impact of the selective use of plasma from male donors in the American Red Cross. Transfusion 2007;47:599-607.
6 Köster F, Wullenweber U, Weinstock C, Madla W, Markovic A: The prevalence of leucocyte antibodies in female blood donors with multiple pregnancies in history. Transfus Med Hemother 2007;34 (suppl 1)21.

7 Reil A, Günay S, Beckmann D, Bux J: Prospective screening for leucocyte antibodies an implication for TRALI prevention. Transfus Med Hemother 2007;34(suppl 1):21.

8 Sachs U, Link E, Bein G: Screening of multiparous women to avoid TRALI. A single center experience. Transfus Med Hemother 2007;34(suppl 1):22.

9 Announcements to the Paul-Ehrlich-Institute (PEI) according \$21 TFG. www.pei.de.

10 Kakaiya RM, Rios J, Triulzi DJ, Hillyer CD, Kleinman S, Busch MP, Gottschall JL, Carey PM, Schreiber GB, Schlumpf KS, Nemo G: Blood donations from previously transfused or pregnant donors: A multi-center study to determine the frequency of allo-exposure. Transfusion 2007;47 (suppl):6A.
11 Wahl S: Geburtenverhalten in Deutschland und anderen ausgewählten Ländern. Instititut für Wirtschaft und Gesellschaft (IWG) Bonn e. V., IWGImpulse, Bonn 2003. www.iwg-bonn.de/index.php? $i d=61$.

12 Bux J: Molecular nature of antigens implicated in immune neutropenias. Int J Hematol 2002;76: 399-403.

13 Maślanka K, Michur H, Zupa ska B, Uhrynowska M, Nowak J: Leucocyte antibodies in blood donors and a look back on recipients of their blood components. Vox Sang 2007;92:247-249.

14 Win N, Ranansinghe E, Lucas G: Transfusion associated acute lung injury: a 5-year look-back study. Transfus Med 2002;12:387-389.

15 Toy P, Hollis-Perry KM, Jun J, Nakagawa M: Recipients of blood from a donor with multiple HLA antibodies: a lookback study of transfusion related acute lung injury. Transfusion 2004:44:1683-1688. 